\title{
SEPARABLE GONALITY OF A GORENSTEIN CURVE
}

\author{
Masaaki Homma
}

\section{Introduction}

Just as in the case of smooth curves, an integral projective curve $X$, which may have singular points, of arithmetic genus $g \geq 2$ is said to be hyperelliptic if there is a finite morphism $X \rightarrow \mathbf{P}^{1}$ of degree 2 . However, there is a phenomenon which never happens in the case of smooth hyperelliptic curves; that is, the degree-two morphism may be inseparable. A hyperelliptic curve with this property is said to be of inseparable type. The complete picture of singular hyperelliptic curves can be found in [3].

On the other hand, any (singular or nonsingular) hyperelliptic curve is Gorenstein ([4, Th. 15], [3, (2.2)]), i.e. the dualizing sheaf of the curve is invertible. The purpose of this short note is to give a characterization of hyperelliptic curves of inseparable type in the category of Gorenstein curves in terms of the separable gonality of a curve, which is defined to be the smallest possible degree of a finite separable morphism from the curve to the projective line. Our result should be placed in a more general context of a divisor theory of a Gorenstein curve; however, here we will give a makeshift proof to it.

Throughout this note, we will assume the ground field $K$ to be algebraically closed.

\section{The inequality $k_{s} \leq g+1$}

Let $X$ be an integral projective curve of genus $g$. We will show that there is a finile separable morphism $X \rightarrow \mathbf{P}^{1}$ of degree less than or equal to $g+1$. 
In fact, let us take pairwise distinct $g+1$ smooth points $P_{1}, \cdots, P_{g+1}$ of $X$. Then we have $h^{0}\left(\mathcal{O}_{X}\left(P_{1}+\ldots+P_{g+1}\right)\right) \geq 2$ by the Riemann-Roch theorem. Hence there is a non-constant function $f: X \rightarrow \mathbf{P}^{1}$ whose pole divisor $(f)_{\infty}$ is at most $P_{1}+\cdots+P_{g+1}$. Since $v_{P}(f)=-1$ for any $P \in(f)_{\infty}$, the morphism $f$ is separable.

Therefore we can define the separable gonality $k_{s}=k_{s}(X)$ of $X$ in the way which was mentioned in Introduction.

\section{Main result}

Our theorem is as follows.

Theorem. Lel $X$ be a Gorenstein curve of genus $g \geq 2$. Then

$$
k_{s}(X) \leq g+1 .
$$

Furthermore, equality occurs if and only if $X$ is hyperelliptic of inseparable type.

Proof. The first part of the assertion has been proved in the previous section.

First we will show that

$$
k_{s}(X) \geq g+1
$$

for a hyperelliptic curve $X$ of inseparable type. By definition, there are two particular finite morphisms from $X$ to $\mathbf{P}^{1}$; one, say $x$, is an inseparable morphism of degree 2 and the other, say $y$, is separable of degree $k_{s}$. Since the function field of $X$ is $K(x, y)$, the morphism

$$
(x, y): X \rightarrow \mathbf{P}^{1} \times \mathbf{P}^{1}
$$

is birational onto its image. Hence we have

$$
g \leq(2-1)\left(k_{s}-1\right)
$$

by virtue of Castelnuovo's inequality. 
Next we will show that

$$
k_{s}(X) \leq g
$$

if $X$ is a Gorenstein curve that is hyperelliptic of separable type (i.e. the degreetwo morphism is separable) or nonhyperelliptic. By definition, $k_{s}(X)=2$ if $X$ is hyperelliptic of separable type. Let $X$ be a nonhyperelliptic Gorenstein curve. Then the canonical linear system is very ample ([4, Th. 17], 2, (1.6)], [5, (3.3)]), that is, $X$ can be embedded in $\mathbf{P}^{g-1}$ as a curve of degree $2 g-2$. Hence, by using Bertini's theorem [1, II (8.18) and (8.18.1)], we can find pairwise distinct $2 g-2$ smooth points $P_{1}, \cdots, P_{2 g-2}$ so that

$$
h^{0}\left(\mathcal{O}_{X}\left(P_{1}+\ldots+P_{2 g-2}\right)\right)=g .
$$

Hence $h^{0}\left(\mathcal{O}_{X}\left(P_{1}+\ldots+P_{g}\right)\right) \geq 2$. Therefore we can conclude that $k_{s} \leq g$ by the same argument in Section 1.

Remark. The first part of the statement of Theorem holds without assuming $X$ to be Gorenstein, but the second part does not.

In fact, let us consider the curve $Y_{g}$ obtained from the projective line $\mathbf{P}^{1}$ by replacing the local ring $\mathcal{O}_{\mathbf{P}^{1}, 0}$ by

$$
K+t^{g+1} \mathcal{O}_{\mathbf{P}^{1}, 0}
$$

where $t$ is a uniformizing function on $\mathbf{P}^{1}$ so that $t \mathcal{O}_{\mathbf{P}^{1}, 0}$ is the maximal ideal of $\mathcal{O}_{\mathbf{P}^{1}, 0}$. If $g \geq 2$, then $Y_{g}$ is a non-Gorenstein curve of genus $g$. Looking at the local ring of $Y_{g}$ at 0 , we know that every nonconstant function $Y_{g} \rightarrow \mathbf{P}^{1}$ is of degree greater than $g$. Hence $Y_{g}$ is a nonhyperelliptic curve with $k_{s}\left(Y_{g}\right)=g+1$.

Acknowledgment. This work was done while the author was visiting IMPA under JSPS-ABC exchange program. He is deeply grateful to IMPA for their hospitality. 


\section{References}

[1] Hartshorne, R., Algebraic geometry, Graduate texts in Mathematics 52, Springer-Verlag, New York, (1977)

[2] Hartshorne, R., Generalized divisors on Gorenstein curves and a theorem of Noether, J. Math. Kyoto Univ. 26 (1986), 375-386.

[3] Homma, M. Singular hyperelliptic curves, preprint, 1996

[4] Rosenlicht, M., Equivalence relations on algebraic curves, Ann. of Math. 56 (1952), 169-191.

[5] Stöhr, K. O., On the poles of regular differentials of singular curves, Bol. Soc. Bras. Mat. 24 (1993), 105-136.

Department of Mathematics

Kanagawa University

Rokkakubashi, Kanagawa-ku

Yokohama 221 Japan

E-mail homma@cc.kanagawa-u.ac.jp 